\title{
A 6-month "self-monitoring" lifestyle modification with increased sunlight exposure modestly improves vitamin D status, lipid profile and glycemic status in overweight and obese Saudi adults with varying glycemic levels
}

\author{
Nasser M Al-Daghri ${ }^{1,2^{*}}$, Hanan Alfawaz ${ }^{1,3}$, Naji J Aljohani ${ }^{1,4}$, Yousef Al-Saleh ${ }^{1,5}$, Kaiser Wani $^{1,2}$, \\ Abdullah M Alnaami ${ }^{1,2}$, Mohammad Alharbi ${ }^{6}$ and Sudhesh Kumar ${ }^{7}$
}

\begin{abstract}
Background: The over-all age-adjusted prevalence of diabetes mellitus type 2 (DMT2) in Saudi Arabia is unprecedented at 31\%. Aggressive measures should be done to curb down increasing incidence. In this prospective 6-month study we aim to determine whether a self-monitoring, life-style modification program that includes increased sunlight exposure confer improvement in vitamin D status and health benefits among adult Saudi overweight and obese patients with varying glycemic status.

Methods: A total of 150 overweight and obese Saudi adults with varying glycemic status aged 30-60 years were included in this study. They were divided into 3 groups (Non-DMT2, Pre-diabetes and DMT2). Baseline anthropometrics and blood glucose were taken at baseline and after 6 months. Fasting blood sugar, lipid profile, calcium, albumin and phosphate were measured routinely. Serum $25(\mathrm{OH})$ vitamin D was measured using standard assays. Within the time period they were instructed to reduce total intake of fat, increased fiber intake and increase sun exposure.

Results: In all groups there was a significant improvement in vitamin D levels as well as serum triglycerides, LDL- and total cholesterol. However, a significant increase in serum glucose levels was noted in the non-DMT2 group, and a significant decrease in HDL-cholesterol in both non-DMT2 and pre-diabetes group. In the pre-diabetes group, 53.2\% were able to normalize their fasting blood levels after 6 months, with 8.5\% reaching the DMT2 stage and 38.3\% remaining pre-diabetic. In all groups there was a significant increase in the prevalence of hypertension.

Conclusion: Improving vitamin D status with modest lifestyle modifications over a short-period translates to improvement in lipid profile except HDL-cholesterol among overweight and obese Saudi adults, but not BMI and blood pressure. Findings of the present study merit further investigation as to whether full vitamin D status correction can delay or prevent onset of DMT2.
\end{abstract}

Keywords: Vitamin D, Self-monitoring, Diabetes mellitus, Pre-diabetes, Obesity

\footnotetext{
* Correspondence: aldaghri2011@gmail.com

${ }^{1}$ Prince Mutaib Chair for Biomarkers of Osteoporosis, Biochemistry

Department, King Saud University, PO Box, 2455, Riyadh 11451, Saudi Arabia

2Biomarkers Research Program, Biochemistry Department, College of Science,

King Saud University, Riyadh, Saudi Arabia

Full list of author information is available at the end of the article
} 


\section{Background}

If the prevalence of diabetes mellitus type 2 (DMT2) continues to increase at the current rate, the global burden of this disease will swell between from 171 million in 2000, to 366 million patients in 2030 [1]. Furthermore, healthcare expenditures on DMT2 alone will skyrocket from US\$376 billion in 2010 to US\$490 billion in 2030 [2]. The Middle East region has not been spared from this scourge and currently is among those worst-hit [1]. This global epidemic, shared by both industrialized and developing countries, has stimulated increased public awareness of the disease, the identification of risk factors and the knowledge that DMT2 can be delayed and, even better, prevented [3-5]. Recognition of the importance of glycemic control in the prevention of the complications and morbidity of DMT2 has led to worldwide campaigns for modifications in lifestyle and an intensive search for better anti-diabetes medications [6-8].

Harboring pre-diabetes, or state of intermediate hyperglycemia (plasma glucose levels higher than normal, but lower than the threshold of DM), puts an individual at high risk not only for developing DM but associated complications as well. Among the Gulf neighboring countries, the prevalence of pre-diabetes based on several low-moderate sample-sized cross-sectional studies is moderately high: 9.0\% in Saudi Arabia [9], 13.8\% in Qatar [10], 40.9\% in Oman [11], and $24.2 \%$ in United Arab Emirates (UAE) [12]. According to an expert from the American Diabetes Association (ADA), almost 70\% of the pre-diabetes population will eventually develop DMT2, and in over a 3- to 5-year period, persons who harbor pre-diabetes have a 5- to 15-fold higher risk of further progressing to DMT2 as opposed to their normoglycemic counterparts $[13,14]$. Having mentioned such and considering the global economic burden of DMT2, not to mention the perpetual obstacles to deliver high-quality standard care to DMT2 patients, the challenge to fight DMT2 through prevention is a compelling case worthy of attention and enormous investigation.

Fortunately during the recent years, there has been accumulating evidence from various interventional studies on the beneficial effects of improving vitamin D status among patients with DMT2 and the full metabolic syndrome [15-17]. This suggests some important clinical implications among patients at risk of developing DMT2. However, there is limited information on the role of improving vitamin D status among patients with prediabetes, specifically in this region where the prevalence of both DMT2 and vitamin D deficiency are high. Having mentioned such, the present interventional study aims to determine whether a self-monitoring, life-style modification program that includes increased sunlight exposure confer improvement in vitamin D status and health benefits among adult Saudi overweight and obese patients with varying glycemic status.

\section{Methods}

\section{Site and duration of the study}

This is a multi-center, interventional study to be conducted at the primary care centers of the RIYADH Cohort, a capital-based screening program involving more than 17000 Saudi subjects randomly selected to participate in epidemiologic studies conducted by the Biomarkers Research Program (BRP) of King Saud University (KSU) and the Ministry of Health (MOH), Riyadh, Saudi Arabia.

\section{Subjects}

A total of 200 Saudi adult males and females aged 30-60 years old with unknown DM status and varying body mass indices (BMI) were initially recruited to take part in this 6-month interventional study. These subjects were recruited from the community participants of the Saudi Diabetes Charity Foundation based in Riyadh, Saudi Arabia. Out of the 200 subjects, 50 dropped out for various reasons (non-compliance, change of medication, lost to follow up, etc.). They were divided into 3 groups based on baseline fasting blood glucose (FBG) levels: NonDMT2 group (FBG $<5.6 \mathrm{mmol} / \mathrm{l})$, Pre-DMT2 group (FBG 5.6-6.9 mmol/l) and DMT2 group (FBG $\geq 7.0 \mathrm{mmol} / \mathrm{l}$ ). Non-consenting subjects, pregnant subjects, and anyone with complications such as renal, neurologic, hepatic and pulmonary diseases were excluded as well as acute conditions that require immediate medical attention. The study was performed in accordance with the the ethical principles in the Declaration of Helsinki as well as with the ICH Note for Guidance on Good Clinical Practice. Approval from the ethics committee of the College of Science, KSU was sought prior to study commencement.

\section{Clinical assessment}

Baseline anthropometric measurements were noted by the assigned and registered primary care physician and nurse. These included height $(\mathrm{cm})$ and weight $(\mathrm{kg})$ from which BMI was calculated [weight $(\mathrm{kg}) /$ height $\left(\mathrm{m}^{2}\right)$ ]. Blood pressure $(\mathrm{mmHg})$ was taken twice after 30 minutes rest using the conventional mercurial sphygmomanometer and the average was recorded. All measurements were repeated after 6 months.

\section{Laboratory parameters}

All subjects were requested to submit an over-night fasting blood samples from which the different metabolic parameters were assessed. Serum FBG, complete lipid profile, serum albumin, calcium and phosphate were determined using routine laboratory methods. Serum $25(\mathrm{OH})$ vitamin D was measured using an enzyme-linked immunosorbent assay (ELISA) (IDS Ltd., Boldon Colliery, Tyne \& 
Wear, UK). The inter- and intra-assay variabilities were $5.3 \%$ and $4.6 \%$. All measurements were done in a DEQAS(Vitamin D External Quality Assessment Scheme) participating laboratory, the Biomarkers Research Program (BRP) of King Saud University, Riyadh, KSA.

\section{Lifestyle intervention}

All subjects were oriented and educated about lifestyle modifications from successful intervention programs done elsewhere [18,19], advised and followed-up regularly during specified visits. In brief, the intervention program has specific goals which include weight reduction of $5 \%$ or more, total intake of fat to $<30 \%$ of energy consumed, intake of saturated fat $<10 \%$ of energy consumed, increased fiber intake to at least $15 \mathrm{~g} / 1000 \mathrm{kcal}$ and moderate exercise for at least 30 minutes per day (30 minute walk, 5 times a week). Some of these goals are exemplified as taken from the Diabetes Prevention Program (DPP): eat smaller portions; drink more water and little or no sugar-containing beverages each day; choose leaner cuts of beef, eat white meat of turkey and chicken more often, eat fish high in omega-3; fatty acids, and no more than 340 gram per week; increase intake of whole fruits and vegetables; choose whole grains like rolled oats, barley, bran, and $100 \%$ whole grain bread instead of refined, processed carbohydrates like baked products made with white flour; choose low or no fat dairy products; use unsaturated vegetable oils that are liquid at room temperature like olive, canola, peanut, safflower, sunflower, corn, soybean and cottonseed oils, and use soft-tub, squeeze or spray margarine; eat at regular mealtimes; use low-fat food preparation (grilling, broiling, boiling, steaming, etc....); eat breakfast and reduce frequency of eating out, especially in fast food restaurants [20].

The orientation and intervention were conducted by a certified nutritionist, physician, nurse and physical therapists, who were also the same team that reinforced instructions to the subjects on a regular basis. In addition, they were advised to increase sunlight exposure during the entire 6 months of the study. In all subjects, "self-monitoring" was introduced, and they were subjected to regular calls from the investigator to keep track of the progress.

\section{Data analysis}

Collected data was analyzed using SPSS version 16.5 for windows (Chicago, Illinois, USA). Frequencies were presented in percent (\%) cases and Chi-square was used to determine differences. Paired T-test was done to determine intervention effects in anthropometric and metabolic parameters at baseline and after 6 months. Bivariate correlation coefficients between the variables were determined. $P$-value $\leq 0.05$ will be considered significant.

\section{Results}

Table 1 highlights the characteristics of all subjects and the non-diabetic group. In all subjects, no significant changes were observed in BMI, blood pressure and fasting blood glucose overtime. Serum triglycerides, total cholesterol, LDL- and HDL-cholesterol significantly decreased after 6 months compared to baseline ( $p$-values $0.048,5.3 \times 10^{-6}, 2.5 \times 10^{-5}$ and $3.0 \times 10^{-4}$, respectively). On the other hand, baseline serum calcium and albumin were noted to be significantly higher as compared to 6 months ( $p$-values $1.1 \times 10^{-7}$ and 0.026 , respectively. There was also a significant increase in levels of circulating $25(\mathrm{OH})$ vitamin $\mathrm{D}$ from baseline to 6 months ( $p$-value $\left.3.3 \times 10^{-4}\right)$. In the non-diabetic group, baseline BMI and blood pressure remained almost unchanged after 6 months follow-up. However, a significant increase in fasting serum glucose was noted after 6 months ( $p$-value 0.0002$)$. With regards to lipids, changes in baseline serum triglycerides, total and LDL-cholesterol were not significant after 6 months follow-up. However, follow-up HDL-cholesterol was significantly higher as compared to baseline levels ( $p$-value 0.0002). Baseline serum albumin levels significantly increased after 6 months as well as baseline serum $25(\mathrm{OH})$ vitamin D ( $p$-values 0.004, 0.004 respectively).

Table 2 shows the metabolic changes in both the DMT2 group and the pre-diabetes group. In both groups, no significant improvements were observed in BMI, blood pressure, serum glucose and triglycerides over time. However, and also in both groups, a significant decrease was observed in serum total and LDLcholesterol (DMT2 group p-values 0.0004 and $7.6 \times 10^{-5}$; pre-DM group $p$-values 0.002 and 0.017 , respectively. Changes in HDL-cholesterol were noted only in the preDM group, in which baseline levels were significantly higher than after 6 months ( $p$-value 0.009). Serum calcium levels on the other hand significantly increased from baseline to 6 months in both groups (DMT2 group $p$-value 0.015 ; pre-DM group $=0.012$ ). Lastly, improvements in 25 $(\mathrm{OH})$ vitamin $\mathrm{D}$ levels were noted only in the pre-DM group, with borderline significance ( $p$-value 0.05 ).

Table 3 shows the associations of serum $25(\mathrm{OH})$ vitamin D to baseline anthropometric and metabolic parameters as well as after 6 months. In all subjects, only serum calcium was positively associated with $25(\mathrm{OH})$ vitamin $\mathrm{D}$ levels $(\mathrm{R}=0.19 ; p$-value $<0.05)$. This was also true for the pre-DM group $(\mathrm{R}=0.27 ; p$-value 0.05$)$ while for the non-DMT2 group, serum calcium was significantly associated with $25(\mathrm{OH})$ vitamin $\mathrm{D}$ only at baseline) $\mathrm{R}=0.27$; $p$-value $<0.05)$. With regards to lipids, serum triglycerides and total cholesterol were both negatively and significantly associated with $25(\mathrm{OH})$ vitamin D levels, but only in the pre-DM group at baseline $(R=-0.27$ and -0.29 , respectively). In the DMT2 group, only serum phosphate was significantly associated with $25(\mathrm{OH})$ vitamin D levels 
Table 1 Anthropometric and metabolic changes of all subjects and non-DM group over time

\begin{tabular}{|c|c|c|c|c|c|c|}
\hline & & All subjects & & & n-DMT2 grou & \\
\hline & Baseline & 6 months & P-value & Baseline & 6 months & P-value \\
\hline$M / F$ & & $37 / 113$ & & & $15 / 51$ & \\
\hline Age (years) & & $44.78 \pm 0.93$ & & & $39.8 \pm 1.44$ & \\
\hline BMI $\left(\mathrm{kg} / \mathrm{m}^{2}\right)$ & $32.3 \pm 0.5$ & $32.2 \pm 0.5$ & NS & $32.6 \pm 0.8$ & $32.5 \pm 0.8$ & NS \\
\hline Systolic blood pressure (mmHg) & $115.7 \pm 1.7$ & $115.4 \pm 1.8$ & NS & $115.4 \pm 2.4$ & $114.6 \pm 2.5$ & NS \\
\hline Diastolic blood pressure (mmHg) & $84.8 \pm 2.2$ & $85.1 \pm 24.7$ & NS & $81.2 \pm 3.3$ & $82.6 \pm 3.3$ & NS \\
\hline Glucose (mmol/l) & $6.1 \pm 0.2$ & $6.0 \pm 0.2$ & NS & $4.6 \pm 0.1$ & $5.2 \pm 0.1$ & 0.0002 \\
\hline Triglycerides (mmol/l) & $1.7 \pm 0.07$ & $1.6 \pm 0.06$ & 0.048 & $1.62 \pm 0.1$ & $1.47 \pm 0.08$ & NS \\
\hline Total cholesterol (mmol/l) & $4.7 \pm 0.09$ & $4.3 \pm 0.08$ & $5.3 \times 10^{-6}$ & $4.6 \pm 0.13$ & $4.3 \pm 0.11$ & NS \\
\hline HDL-cholesterol (mmol/l) & $1.1 \pm 0.03$ & $0.87 \pm 0.05$ & $3.0 \times 10^{-4}$ & $1.1 \pm 0.04$ & $0.84 \pm 0.07$ & 0.002 \\
\hline LDL-cholesterol (mmol/l) & $3.5 \pm 0.11$ & $3.1 \pm 0.09$ & $2.5 \times 10^{-5}$ & $3.3 \pm 0.14$ & $3.06 \pm 0.12$ & NS \\
\hline Calcium (mmol/l) & $2.1 \pm 0.01$ & $2.2 \pm 0.01$ & $1.1 \times 10^{-7}$ & $2.07 \pm 0.02$ & $2.23 \pm 0.02$ & 0.0008 \\
\hline Corrected calcium (mmol/l) & $2.2 \pm 0.01$ & $2.3 \pm 0.01$ & $7.3 \times 10^{-8}$ & $2.17 \pm 0.02$ & $2.28 \pm 0.02$ & 0.001 \\
\hline Phosphate (mmol/l) & $1.08 \pm 0.05$ & $1.11 \pm 0.02$ & NS & $1.08 \pm 0.12$ & $1.09 \pm 0.02$ & NS \\
\hline Albumin $(\mathrm{g} / \mathrm{l})$ & $36.4 \pm 0.46$ & $37.6 \pm 0.37$ & 0.026 & $35.2 \pm 0.68$ & $37.6 \pm 0.6$ & 0.004 \\
\hline 25-OH D (nmol/l) & $45.7 \pm 2.2$ & $55.8 \pm 2.7$ & $3.3 \times 10^{-4}$ & $42.3 \pm 2.8$ & $53.6 \pm 3.6$ & 0.004 \\
\hline
\end{tabular}

Note: Data presented as mean \pm standard error; NS - not significant; significance at $p<0.05$.

$(\mathrm{R}=0.34 ; p$-value $<0.05)$, and this was after 6 months. The rest of the associations were non-contributory.

Lastly, Table 4 shows the changes in the cardiometabolic risk factors from baseline and after 6 months. In Figure 1, the most notable changes were observed in the glycemic status, in which all those who were non-DMT2 at baseline dropped to $70 \%$ after follow-up, with $25.4 \%$ under the category of pre-DM and $4.5 \%$ classified under
DMT2. In the baseline pre-DM group, $53.2 \%$ were able to normalize their fasting blood levels after 6 months, with $8.5 \%$ reaching the DMT2 stage and 38.3\% remaining pre-diabetic. Finally in the baseline DMT2 group, $13.2 \%$ were able to normalize their fasting blood glucose after 6 months, with $36.8 \%$ also improving but still in the prediabetes stage and $50 \%$ remaining well within the cut-off of DMT2. In all groups there was a significant increase in the

Table 2 Anthropometric and metabolic changes of DM and pre-diabetes group over time

\begin{tabular}{|c|c|c|c|c|c|c|}
\hline & \multicolumn{3}{|c|}{ DMT2 group } & \multicolumn{3}{|c|}{ Pre-diabetes group } \\
\hline & Baseline & 6 months & P-value & Baseline & 6 months & P-value \\
\hline$M / F$ & & $8 / 29$ & & & $14 / 33$ & \\
\hline Age (years) & & $47.69 \pm 1.45$ & & & $48.85 \pm 1.46$ & \\
\hline BMI $\left(\mathrm{kg} / \mathrm{m}^{2}\right)$ & $31.8 \pm 1.0$ & $32.1 \pm 0.9$ & NS & $32.2 \pm 0.97$ & $32.0 \pm 0.92$ & NS \\
\hline Systolic blood pressure (mmHg) & $115.7 \pm 3.6$ & $116.2 \pm 4.4$ & NS & $116.3 \pm 3.5$ & $115.8 \pm 3.2$ & NS \\
\hline Diastolic blood pressure $(\mathrm{mmHg})$ & $90.8 \pm 4.9$ & $89.7 \pm 5.6$ & NS & $85.3 \pm 3.5$ & $85.0 \pm 3.8$ & NS \\
\hline Glucose (mmol/l) & $8.6 \pm 0.4$ & $7.8 \pm 0.4$ & NS & $6.1 \pm 0.05$ & $5.8 \pm 0.17$ & NS \\
\hline Triglycerides (mmol/l) & $2.0 \pm 0.17$ & $1.9 \pm 0.12$ & NS & $1.7 \pm 0.12$ & $1.6 \pm 0.11$ & NS \\
\hline Total cholesterol (mmol/l) & $5.0 \pm 0.21$ & $4.3 \pm 0.17$ & 0.0004 & $4.8 \pm 0.14$ & $4.3 \pm 0.13$ & 0.002 \\
\hline HDL-cholesterol (mmol/l) & $0.98 \pm 0.06$ & $0.94 \pm 0.11$ & NS & $1.07 \pm 0.05$ & $0.86 \pm 0.08$ & 0.009 \\
\hline LDL-cholesterol (mmol/l) & $3.8 \pm 0.3$ & $2.97 \pm 0.2$ & $7.6 \times 10^{-5}$ & $3.6 \pm 0.18$ & $3.1 \pm 0.16$ & 0.017 \\
\hline Calcium (mmol/l) & $2.1 \pm 0.03$ & $2.2 \pm 0.04$ & 0.015 & $2.09 \pm 0.02$ & $2.18 \pm 0.02$ & 0.012 \\
\hline Corrected calcium (mmol/l) & $2.2 \pm 0.02$ & $2.3 \pm 0.03$ & 0.002 & $2.15 \pm 0.02$ & $2.23 \pm 0.02$ & 0.005 \\
\hline Phosphate (mmol/l) & $1.08 \pm 0.03$ & $1.1 \pm 0.04$ & NS & $1.08 \pm 0.02$ & $1.08 \pm 0.02$ & NS \\
\hline Albumin (g/l) & $37.4 \pm 0.9$ & $38.1 \pm 0.7$ & NS & $37.4 \pm 0.87$ & $37.3 \pm 0.52$ & NS \\
\hline 25-OH D (nmol/l) & $46.8 \pm 3.6$ & $54.7 \pm 4.8$ & NS & $49.3 \pm 5.2$ & $60.7 \pm 5.8$ & 0.05 \\
\hline
\end{tabular}

Note: Data presented as mean \pm standard error; NS - not significant; significance at $p<0.05$. 
Table 3 Associations of $25(\mathrm{OH})$ vitamin $D$ to metabolic parameters according to groups

\begin{tabular}{|c|c|c|c|c|c|c|c|c|}
\hline & \multicolumn{2}{|c|}{ ALL subjects $N=150$} & \multicolumn{2}{|c|}{ Non-DMT2 group $\mathrm{N}=67$} & \multicolumn{2}{|c|}{ Pre-DM group $\mathrm{N}=47$} & \multicolumn{2}{|c|}{ DMT2 group $\mathrm{N}=37$} \\
\hline & Baseline & 6 months & Baseline & 6 months & Baseline & 6 months & Baseline & 6 months \\
\hline BMI $\left(\mathrm{kg} / \mathrm{m}^{2}\right)$ & -0.10 & -0.11 & 0.06 & 0.07 & -0.19 & $-0.34^{*}$ & -0.06 & -0.03 \\
\hline Systolic blood pressure (mmHg) & 0.10 & -0.04 & 0.05 & 0.00 & 0.01 & -0.02 & 0.21 & -0.20 \\
\hline Diastolic blood pressure $(\mathrm{mmHg})$ & 0.03 & -0.05 & 0.08 & -0.10 & -0.06 & -0.18 & -0.04 & 0.16 \\
\hline Glucose $(\mathrm{mmol} / \mathrm{l})$ & 0.07 & 0.15 & 0.05 & 0.04 & 0.008 & 0.23 & 0.02 & 0.20 \\
\hline Triglycerides (mmol/l) & -0.04 & -0.04 & 0.04 & 0.04 & -0.11 & $-0.27^{*}$ & -0.14 & 0.18 \\
\hline Total cholesterol (mmol/l) & -0.10 & -0.15 & -0.02 & -0.10 & -0.20 & $-0.29^{*}$ & -0.11 & -0.03 \\
\hline HDL-cholesterol (mmol/l) & -0.06 & -0.05 & 0.05 & -0.13 & -0.28 & -0.02 & 0.09 & 0.12 \\
\hline LDL-cholesterol (mmol/l) & -0.05 & -0.14 & 0.02 & -0.06 & -0.05 & -0.22 & -0.15 & -0.14 \\
\hline Calcium (mmol/l) & 0.05 & $0.19^{*}$ & $0.27^{*}$ & 0.23 & -0.04 & $0.27^{*}$ & -0.32 & 0.10 \\
\hline Corrected calcium (mmol/l) & -0.02 & 0.15 & 0.20 & 0.17 & -0.23 & 0.22 & -0.11 & 0.09 \\
\hline Phosphate (mmol/l) & -0.02 & 0.07 & 0.19 & 0.16 & -0.23 & -0.19 & 0.00 & $0.34^{*}$ \\
\hline Albumin $(\mathrm{g} / \mathrm{l})$ & 0.07 & 0.09 & 0.12 & 0.12 & 0.13 & 0.06 & -0.19 & 0.08 \\
\hline
\end{tabular}

Note: Data presented as coefficient (R); ${ }^{*}$ denotes significance at $p<0.05$.

prevalence of hypertension, while a significant decrease in the prevalence of hypertriglyceridemia was observed in the pre-DM group. Regardless of the glycemic status at baseline, there was a significant improvement in vitamin D status, with baseline vitamin D deficiency dropping significantly in all groups, but still high over-all (Table 4).

\section{Discussion}

The significant finding of the present interventional study was that the over-all improvement in the vitamin $\mathrm{D}$ status of the cohort after 6 months, as evidenced by the significant decrease in mean vitamin $\mathrm{D}$ levels and vitamin $\mathrm{D}$ deficiency prevalence, led to modest improvements at most, in the lipid profile that includes triglycerides, LDL- and total cholesterol, with no improvements observed in BMI and blood pressure. Furthermore, vitamin D status was not associated with majority of the metabolic parameters measured, with only triglycerides showing a positive association among patients with prediabetes and serum calcium in all subjects which was expected, considering that vitamin $\mathrm{D}$ is the main regulator of calcium homeostasis. This lack of promising clinical benefits highlights several caveats in the intervention program, particularly the "self-monitoring" strategy that the investigators employed. Nevertheless, interventional studies done elsewhere among patients with pre-diabetes also observed that raising vitamin D levels either through supplementation for 3 months one year had no effect on insulin sensitivity, pathophysiology of pre-diabetes and development of diabetes [21,22].

Another possible explanation for the lack of profound clinical improvement was that despite the significant improvement of vitamin D status across all groups, the BMI status remained constant, which is the main target of successful programs designed to reduce diabetes risk. The biggest and most convincing documentation for the risk-reducing benefits of lifestyle modification in the USA were studies from the Diabetes Prevention Program (DPP), which apparently was also the first randomized trial to compare lifestyle and a pharmacologic intervention to placebo [20]. Based on the DPP plan, weight reduction was the most powerful predictor of reduced diabetes incidence with an over-all $16.0 \%$ risk reduction for every $1 \mathrm{~kg}$ of reduced weight [23]. Nevertheless, people who achieved physical exercise instead of weight loss also experienced significant reduction in diabetes risk by as much as $44 \%$. Modifications in physical activity and reduction of calories from dietary fat predicted

Table 4 Changes in risk factors over time

\begin{tabular}{|c|c|c|c|c|c|c|c|c|c|}
\hline & \multicolumn{3}{|c|}{ Non-DMT2 group $\mathrm{N}=67$} & \multicolumn{3}{|c|}{ Pre-DM group $\mathrm{N}=47$} & \multicolumn{3}{|c|}{ DMT2 group $N=37$} \\
\hline & Baseline & 6 months & P-value & Baseline & 6 months & P-value & Baseline & 6 months & P-value \\
\hline Obesity & 64.7 & 64.7 & NS & 64.3 & 61.9 & NS & 64.7 & 67.6 & NS \\
\hline Hypertension & 23.6 & 26.0 & 0.006 & 21.4 & 34.3 & 0.0001 & 33.3 & 51.6 & 0.008 \\
\hline Hypertriglyceridemia & 32.4 & 32.4 & NS & 46.8 & 38.3 & 0.04 & 48.6 & 52.6 & NS \\
\hline Vitamin D deficiency & 67.6 & 52.2 & 0.009 & 55.3 & 52.2 & 0.008 & 62.2 & 48.6 & 0.04 \\
\hline
\end{tabular}

Note: Data presented as percentages (\%); NS - not significant; significance at $p<0.05$. 


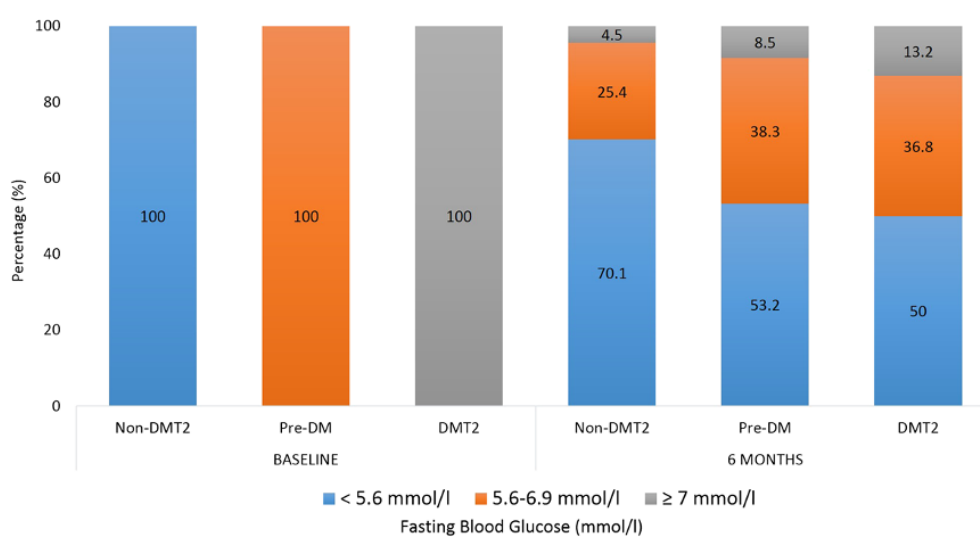

Figure 1 Prevalence of fasting glycemic states at baseline and after 6 months in non-DMT2, Pre-DM and DMT2 groups.

weight loss, and this weight loss translated to lower diabetes risk, further confirming the premise that intercessions to decrease diabetes risk should target weight reduction especially in the overweight and obese population. It is worthy to note however that despite the lack of significant BMI loss, the glycemic status of the DMT2 group improved, with $50 \%$ of the subjects having lower fasting blood glucose levels as compared to their baseline levels. This however is overshadowed by the control group, who, in sharp contrast, had significantly higher mean levels of serum glucose after the intervention.

Another finding worthy of mention is the significant decrease in HDL-cholesterol in all groups despite improvements in the rest of the lipid profile. This finding is in line with several recently published studies questioning whether vitamin D status correction, at least among those who are vitamin $\mathrm{D}$ deficient, have no meaningful clinical changes in lipid concentrations, if not negative effect among those who were not vitamin D deficient [24,25]. This may have been the case in the present cohort, where vitamin D sufficiency is present within $30-40 \%$ across groups. Low HDL-cholesterol is already the most common metabolic syndrome component among Saudis with $90 \%$ of adults harboring such condition [26], and if previous claims are true on the negative effects on lipids on those patients who are not vitamin $\mathrm{D}$ deficient, then further investigations are needed to target only those who are most at risk for vitamin D deficiency, and exclude those whose vitamin D status are normal. Emerging indices for insulin resistance such as visceral adiposity index (VAI) are also suggested to be included in future studies to probe specific improvements outside conventional parameters used in the present study [27].

The present study has several caveats and findings should be interpreted with caution. It may have been underpowered with a short duration of intervention. The method of "self-monitoring" have made the results less beneficial but still favorable, since compliance and adherence is highly linked to the success of the program. Season should have been taken into consideration since it affects vitamin D status in the region [28]. Nevertheless the study has merits in being the first interventional study to observe the effects of sub-optimal vitamin D status replenishment in overweight and obese adults with varying degrees of glycemia in the Middle East.

\section{Conclusion}

In summary, self-monitoring lifestyle modification coupled with increased sun exposure for 6 months improved, but not corrected vitamin D status, with no immediate impact in BMI, blood pressure and glucose levels among Saudi overweight and obese patients with pre-diabetes and DMT2. Significant improvements were most notable in lipid profile, with the exception of HDL-cholesterol, which decreased in all groups. Further interventional studies with a cohort exclusively to those with vitamin D deficiency and a more aggressive approach such as full vitamin D correction through supplementation in the implementation of lifestyle modifications is warranted to elicit a more favorable clinical improvement.

\section{Competing interests}

The authors have no conflict of interest to disclose related to this study.

\section{Authors' contributions}

NMA and NJA conceived the study, oversaw study execution, and contributed to manuscript writing. HA, MA and YA recruited subjects and collected data. KW and AMA recruited subjects and analyzed samples. NMA performed data analysis and AMA wrote the manuscript. SK reviewed/edited the final version of the manuscript. All authors approved the final manuscript.

\section{Acknowledgements}

The authors are grateful to the subjects who participated in the study and the research team of Prince Mutaib Chair for Biomarkers of Osteoporosis (PMCO) in King Saud University, Riyadh, KSA for the technical support.

\section{Funding}

This study has been funded by the National Plan for Science and Technology (NPST) [Grant number 12-MED2881-02] and supported by the Diabetes Charity Foundation in Riyadh, Saudi Arabia. 


\section{Author details}

${ }^{1}$ Prince Mutaib Chair for Biomarkers of Osteoporosis, Biochemistry

Department, King Saud University, PO Box, 2455, Riyadh 11451, Saudi Arabia. ${ }^{2}$ Biomarkers Research Program, Biochemistry Department, College of Science, King Saud University, Riyadh, Saudi Arabia. ${ }^{3}$ Department of Food Science and Nutrition, College of Food Science and Agriculture King Saud University, Riyadh, Saudi Arabia. ${ }^{4}$ Specialized Diabetes and Endocrine Center, King Fahad Medical City, Faculty of Medicine, King Saud bin Abdulaziz University for Health Sciences, Riyadh 11525, Saudi Arabia. ${ }^{5}$ College of Medicine, King Saud bin Abdulaziz University for Health Sciences, Riyadh 11461, Saudi Arabia. ${ }^{6}$ Diabetes Centers and Units Administration, Ministry of Health Riyadh, Saudi Arabia. ${ }^{7}$ Division of Metabolic and Vascular Health, Clinical Sciences Research Institute, University Hospitals Coventry and Warwickshire Trust, Walsgrave, Coventry CV2 2DX, UK.

Received: 30 April 2014 Accepted: 15 May 2014

Published: 26 May 2014

\section{References}

1. Wild S, Rogli G, Green A, Sicree R, King H: Global prevalence of diabetes: estimates for the year 2000 and projections for 2030. Diabetes Care 2004, 27:1047-1053.

2. Zhang P, Zhang X, Brown J, Vistisen D, Sicree R, Shaw J, Nichols G: Global healthcare expenditure on diabetes for 2010 and 2030. Diabetes Res Clin Pract 2010, 87:293-301.

3. Cole A, Nathan DM, Savaria-Porter E, Copeland P, Turchin A, Brunt M, Zusman R, Barrett JA, Wexler D, Case E, McMahon GT, Mort E: An algorithm for the care of type 2 diabetes. Crit Pathw Cardiol 2009, 8:156-165.

4. Rodbard HW, Jellinger PS, Davidson JA, Einhorn D, Garber AJ, Grunberger G, Handelsman Y, Horton ES, Lebovitz H, Levy P, Moghissi ES, Schwartz SS: Statement by an American association of clinical endocrinologists/ American college of endocrinology consensus panel on type 2 diabetes mellitus: an algorithm for glycemic control. Endocr Pract 2009, 15:540-559.

5. Nathan DM, Buse JB, Davidson MB, Ferrannini E, Holman RR, Sherwin R, Zinman B: Medical management of hyperglycemia in type 2 diabetes mellitus: a consensus algorithm for the initiation and adjustment of therapy: a consensus statement from the American diabetes association and the European association for the study of diabetes. Diabetologia 2009, 52:17-30.

6. Warsy AS, El-Hazmi MAF: Diabetes mellitus, hypertension and obesity common multi-factorial disorders in Saudis. East Mediterr Health J 1999 5:1236-1242.

7. Al-Nozha MM, Al-Maatouq MA, Al-Mazrou YY, Al-Harthi SS, Arafah MR, Khalil MZ, Khan NB, Al-Khadra A, Al-Marzouki K, Nouh MS, Abdullah M, Attas O, Al-Shahid MS, Al-Mobeireek A: Diabetes mellitus in Saudi Arabia. Saudi Med J 2004, 25:1603-1610.

8. Yamaoka K, Tango T: Efficacy of lifestyle education to prevent type 2 diabetes. Diabetes Care 2005, 28:2780-2786.

9. Al-Daghri NM, Al-Attas OS, Alokail MS, Alkharfy KM, Yousef M, Sabico SL, Chrousos GP: Diabetes Mellitus type 2 and other chronic non-communicable diseases in the central region, Saudi Arabia (Riyadh cohort 2): a decade of an epidemic. BMC Med 2011, 9:76.

10. Bener A, Zirie M, Janahi IM, Al-Hamaq AO, Musallam M, Wareham NJ: Prevalence of diagnosed and undiagnosed diabetes mellitus and its risk factors in a population-based study in Qatar. Diabetes Res Clin Pract 2009, 84(1):99-106.

11. Ganguly SS, Al-Shafaee MA, Bhargava K, Duttagupta KK: Prevalence of prehypertension and associated cardiovascular risk profiles among prediabetic Omani adults. BMC Public Health 2008, 8:108.

12. Saadi H, Carruthers SG, Nagelkerke N, Al-Maskari F, Afandi B, Reed R, Lukic M, Nicholis MG, Kazam E, Alqawi K, Al-Kaabi J, Leduc C, Sabri S, El-Sadiq M, Elkhumaidi S, Agarwal M, Benedict S: Prevalence of diabetes mellitus and its complications in a population-based sample in Al-Ain, United Arab Emirates. Diabetes Res Clin Pract 2007, 78:369-377.

13. American Association of Diabetes Educators: Primary prevention of type 2 diabetes. Diabetes Educ 2012, 38(1):147-150.

14. Prediabetes and the potential to prevent diabetes. Lancet 2012, 379:2213 [No authors listed]

15. Al-Daghri NM, Alkharfy KM, Al-Othman A, El-Kholie E, Moharram O, Alokail MS, Al-Saleh Y, Sabico S, Kumar S, Chrousos GP: Vitamin D supplementation as an adjuvant therapy for patients with T2DM: an 18-month prospective interventional study. Cardiovascular Diabeto/ 2012, 11:85.

16. Alkharfy KM, Al-Daghri NM, Sabico SB, Al-Othman A, Moharram O, Alokail MS, Al-Saleh Y, Kumar S, Chrousos GP: Vitamin D supplementation in patients with diabetes mellitus type 2 on different therapeutic regimens: a one-year prospective study. Cardiovasc Diabetol 2013, 12:113.

17. Al-Daghri NM, Alkharfy KM, Al-Saleh Y, Al-Attas OS, Alokail MS, Al-Othman A, Moharram O, El-Kholie E, Sabico S, Kumar S, Chrousos GP: Modest reversal of metabolic syndrome manifestations with vitamin D status correction: a 12-month prospective study. Metabolism 2012, 61:661-666.

18. Kosaka K, Noda M, Kuzuya T: Prevention of type 2 diabetes by lifestyle intervention: a Japanese trial in IGT males. Diabetes Res Clin Pract 2005 67:152-162.

19. Tuomilehto J, Lindstrom J, Erikkson JG, Valle TT, Hamalainen H, Illane-Parikka P, Keinanen-Kiukaanniemi S, Laakso M, Louheranta A, Rastas M, Salminen V, Uusitupa M, for the Finnish Diabetes Prevention Study Group: Prevention of type 2 diabetes mellitus by changes in lifestyle among subjects with impaired glucose tolerance. N Engl J Med 2001, 344:1343-1350.

20. The Diabetes Prevention Program Research Group: 10-year follow-up of diabetes incidence and weight loss I the diabetes prevention program outcomes study. Lancet 2009, 374:1677-1686

21. Davidson MB, Duran $P$, Lee ML, Friedman TC: High-dose vitamin D supplementation in people with pre-diabetes and hypovitaminosis D. Diabetes Care 2013, 36:260-266.

22. Harris SS, Pittas AG, Palermo NJ: A randomized, placebo-controlled trial of vitamin D supplementation to improve glycaemia in overweight and obese African Americans. Diabetes Obes Metab 2012, 14:789-794.

23. The Diabetes Prevention Program Research group: The influence of age on the effects of lifestyle modification and metformin in the prevention of diabetes. J Gerontol A Biol Med Sci 2006, 61:1075-1081.

24. Jorde R, Strand Hutchinson M, Kjaergaard M, Sneve M, Grimnes G. Supplementation with high doses of vitamin $D$ to subjects without vitamin $D$ deficiency may have negative effects: pooled data from four intervention trials in tronso. ISRN Endocrinol 2013, 2013:348705

25. Ponda MP, Huang X, Oda MA, Breslow JL, Kaufman HW: Vitamin D may not improve lipid levels: a serial clinical laboratory data study. Circulation 2012, 126(3):270-277.

26. Al-Daghri NM, Al-Attas OS, Alokail MS, Alkharfy KM, Sabico SL, Chrousos GP: Decreasing prevalence of the full metabolic syndrome but a persistently high prevalence of dyslipidemia among Adult Arabs. PLoS One 2010, 5:e12159.

27. Al-Daghri NM, Al-Attas OS, Alokail MS, Alkharfy KM, Charalampidis P, Livadas S, Kollias A, Sabico SL, Chrousos GP: Visceral adiposity index is highly associated with adiponectin values and glycaemic disturbances. Eur J Clin Invest 2013, 43:183-189.

28. Al-Daghri NM, Al-Attas OS, Alokail MS, Alkharfy KM, El-Kholie E, Yousef M, Al-Othman A, Al-Saleh Y, Sabico S, Kumar S, Chrousos GP: Increased vitamin $D$ supplementation recommended during summer season in the gulf region: a counterintuitive seasonal effect in vitamin D levels in adult, overweight and obese Middle Eastern residents. Clin Endocrinol (Oxf) 2012, 76:346-350.

\section{doi:10.1186/1476-511X-13-87}

Cite this article as: Al-Daghri et al:: A 6-month "self-monitoring" lifestyle modification with increased sunlight exposure modestly improves vitamin D status, lipid profile and glycemic status in overweight and obese Saudi adults with varying glycemic levels. Lipids in Health and Disease 2014 13:87. 